\section{Fungal Keratitis Caused by Candida orthopsilosis Successfully Treated with Caspofungin}

\section{Dear Editor,}

Candida orthopsilosis, previously known as a subtype of $C$. parapsilosis, was recently classified as a new strain through gene sequencing [1]. This strain is rare in human infection and had not been reported as a cause of fungal keratitis in Korea.

The first-line antifungal agent for yeast keratitis is topical amphotericin B. However, previous studies have shown that amphotericin B has poor therapeutic effect in treating C. parapsilosis, and voriconazole is considered a good alternative [2]. When voriconazole does not have a therapeutic effect, it is necessary to find another antifungal agent with proper ocular penetration and no systemic toxicity.

In this report, we present a rare case of fungal keratitis caused by $C$. orthopsilosis and demonstrate caspofungin as a new alternative treatment option in fungal keratitis. The institutional review board of Yonsei University College of Medicine approved this study (2020-0121-001).

A 61-year-old female with a past history of vitrectomy for diabetic retinopathy visited our clinic in December 2018 for ocular pain and corneal opacity in the right eye. She had hypertension and diabetes and had previously been using autoserum eyedrops for a corneal abrasion, which repeatedly improved and deteriorated on an occasional basis. At the initial examination, her corrected visual acuity was $2 / 100$ in the right eye. Through slit-lamp examination, a $1.5 \times 1.7-\mathrm{mm}$-sized corneal epi-defect with infiltration and hypopyon were noted (Fig. 1A, 1B). A culture test confirmed the diagnosis of $C$. orthopsilosis, for which an initial treatment of topical voriconazole $1 \%$ was applied every hour. In the first two weeks of treatment, hypopyon and the epi-defect with infiltration were improved

Received: February 21, 2020 Final revision: March 21, 2020

Accepted: March 27, 2020
(Fig. 1C, 1D). An antibiotic sensitivity test demonstrated that the strain was highly sensitive to caspofungin, intermediately sensitive to voriconazole, and less sensitive to amphotericin B. Thus, the patient's treatment was changed to caspofungin $0.5 \%$. After 1 week of treatment with caspofungin, the corneal lesion dramatically improved; it was completely healed after 2 months of treatment, resulting in only mild corneal opacity (Fig. 1E, 1F). No recurrence and ocular toxicity event was reported during the follow-up period (Fig. 1G, 1H).
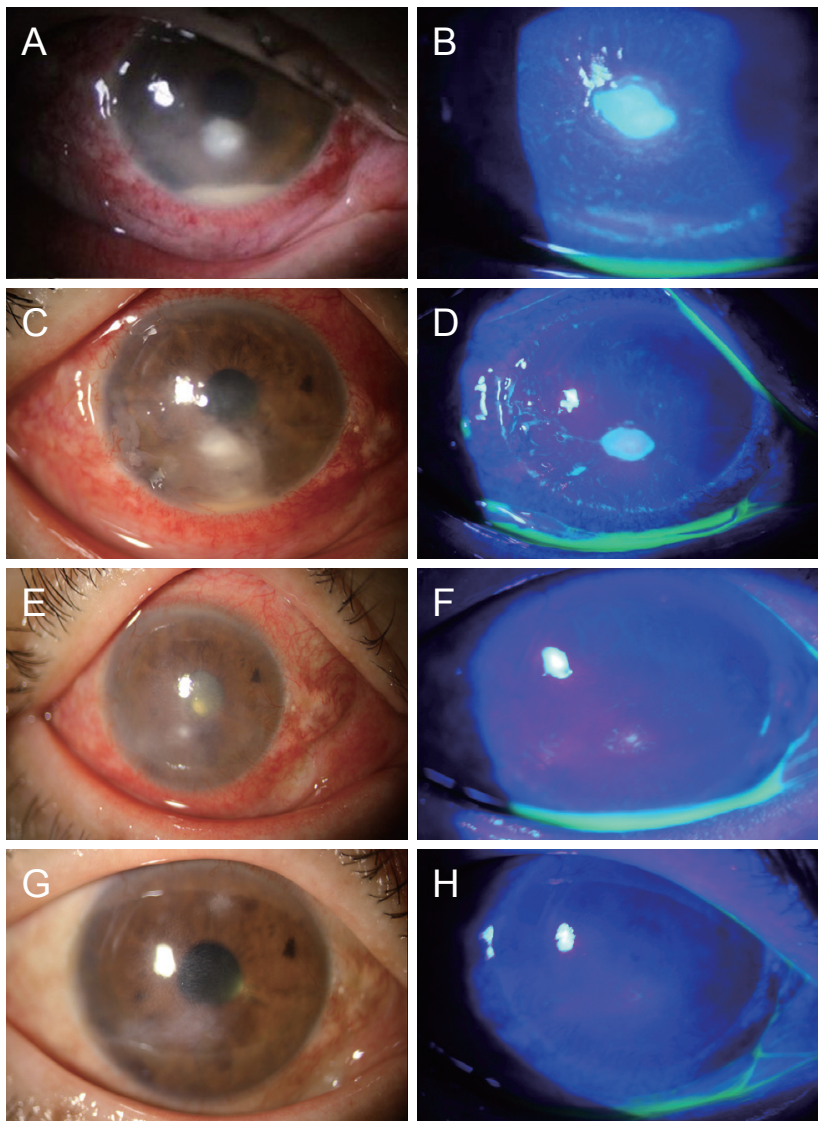

Fig. 1. Serial slit-lamp findings of this case study. (A,B) Slit-lamp photograph at first visit. Corneal epithelial defect and infiltration was located in the center of the cornea and hypopyon was noted. (C,D) Slit-lamp photograph 4 weeks after treatment of voriconazole. Hypopyon and corneal infiltration were slightly improved but still remained. (E,F) Slit-lamp photograph 1 week after treatment of caspofungin. Corneal infiltration was dramatically improved. $(\mathrm{G}, \mathrm{H})$ Slit-lamp photograph 2 months after treatment of caspofungin. Corneal lesion was completely improved, remaining mild corneal opacity. Written informed consent was obtained from the patient. 
Voriconazole is a triazole antifungal agent that inhibits fungal ergosterol biosynthesis through inhibition of 14-alpha-lanosterol demethylation. In addition, amphotericin B is a polyene antifungal agent that binds to ergosterol directly at the cellular membrane, inducing ergosterol sequestration. These two agents commonly cause depletion of ergosterol, disrupting the cell membrane [3].

The present case demonstrated that voriconazole prevented the spread of corneal infiltration by inhibiting growth of the fungus through the mechanism mentioned above. However, because voriconazole had only a fungistatic effect on the strain, it did not completely eradicate the strain. Therefore, we assumed that the strain persisted, and the lesion remained.

Caspofungin is an echinocandin antifungal agent active against Aspergillus and Candida species. Unlike the antifungal agents mentioned above, it acts on the cell wall and inhibits the synthesis of (1,3)-D-glucan, one of the essential elements of cell walls, causing apoptosis [4]. Thus, since it works selectively on fungal cell walls, which do not exist in the human body, it has reduced toxicity in the human body and can be expected to have an effect on strains that are resistant to azole and polyene antifungal agents. Because of these benefits, caspofungin is widely used as a treatment for systemic fungal infection, although it is not yet commonly used as a treatment for ocular infection. However, several recent studies have reported the curative effects of caspofungin on fungal keratitis, and the use of caspofungin as a treatment of fungal keratitis has been increasing [5].

To conclude, we presented a case of fungal keratitis caused by $C$. orthopsilosis, which has not been previously reported in South Korea, and confirmed caspofungin as a new treatment option in cases of voriconazole-resistant fungal keratitis. However, further studies will need to demonstrate the drug efficacy and toxicity of caspofungin as a treatment of fungal keratitis.

Tae Hwan Kim, Ikhyun Jun, Hyung Keun Lee, Kyoung Yul Seo, Tae-Im Kim, Eung Kweon Kim Department of Ophthalmology, Institute of Vision Research, Severance Hospital, Yonsei University College of Medicine, Seoul, Korea

E-mail (Ikhyun Jun): hadesdual@yuhs.ac

\section{Conflict of Interest}

No potential conflict of interest relevant to this article was reported.

\section{Acknowledgements}

This study was supported by the Basic Science Research Program (NRF-2019R1F1A1063311) of the National Research Foundation (NRF) funded by the Ministry of Science, ICT, and Future Planning. The funding organization had no role in the design or conduct of this study.

This study was presented at the 122th annual meeting of the Korean Ophthalmological Society at Seoul, Korea.

\section{References}

1. Tavanti A, Davidson AD, Gow NA, et al. Candida orthopsilosis and Candida metapsilosis spp. nov. to replace Candida parapsilosis groups II and III. J Clin Microbiol 2005;43:284-92.

2. Lee SJ, Lee JJ, Kim SD. Topical and oral voriconazole in the treatment of fungal keratitis. Korean J Ophthalmol 2009;23:46-8.

3. Sponsel W, Chen N, Dang D, et al. Topical voriconazole as a novel treatment for fungal keratitis. Antimicrob Agents Chemother 2006;50:262-8.

4. Neoh CF, Daniell M, Chen SC, et al. Clinical utility of caspofungin eye drops in fungal keratitis. Int J Antimicrob Agents 2014;44:96-104.

5. Hurtado-Sarrio M, Duch-Samper A, Cisneros-Lanuza A, et al. Successful topical application of caspofungin in the treatment of fungal keratitis refractory to voriconazole. Arch Ophthalmol 2010;128:941-2. 